\title{
DETERMINING THE RESPONSE OVER TIME, CURRENT AMPLIFICATION, INPUT AND OUTPUT RESISTANCE OF A PARALLEL-SERIES AMPLIFIER
}

\section{D.C. PUCHIANU}

Valahia University of Targoviste, Faculty of Electrical Engineering, Electronics and Information Technology E-mail: pdantgv@yahoo.com

\begin{abstract}
The study determines the behavior type of the parallel-series amplifier and determines the amplification of the basic amplifier, the input and output resistance, as well as the amplification of the reaction network and the input and output resistance of the reaction network. With the help of the negative reaction, the designer can change the properties of the amplifier. Thus, a dynamic, automatic stability of the amplification system is obtained.
\end{abstract}

Keywords: parallel-series topology, basic amplifier, feedback network

\section{INTRODUCTION}

In negative feedback circuits, the basic idea is that the input quantity in the basic amplifier takes into account the value obtained at the output, so any disturbance or parametric deviation can be corrected, the working frequency band can be extended and the level of noise can be diminished. [1]

The reaction network is chosen only with passive components, usually it is a resistive network, in order to reduce the influence of the parameters dispersion. [2]

In the topology of the parallel-series amplifier on the input, the common quantity is voltage, and the compared quantity is current. At the output the common quantity is the current and the sampled quantity is the voltage. [4] The general topology model is shown in Figure 1, where $a$ is the basic amplification of the amplifier, $f$ is the amplification of the reaction network. Considering the input current $i_{i}$, the output current $i_{0}$ and the load resistance $R_{L}$.

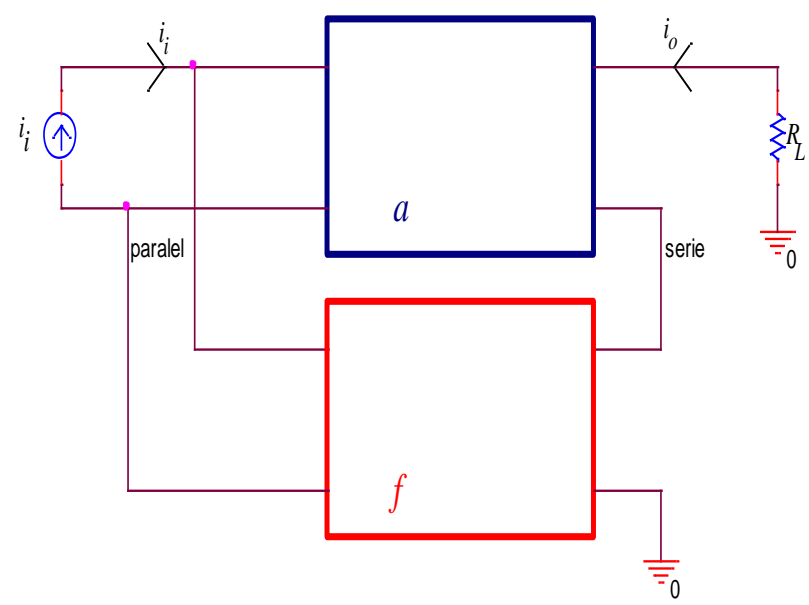

Figure 1. Parallel-series topology
The determination of the current amplification is determined with the circuit shown in Figure 2, where $r_{i}$ is the input resistance of the basic amplifier, $r_{0}$ the output resistance of the basic amplifier, $a i_{\varepsilon}$ the current amplification of the basic amplifier, and $f i_{0}$ is the amplification of the reaction network. [5]

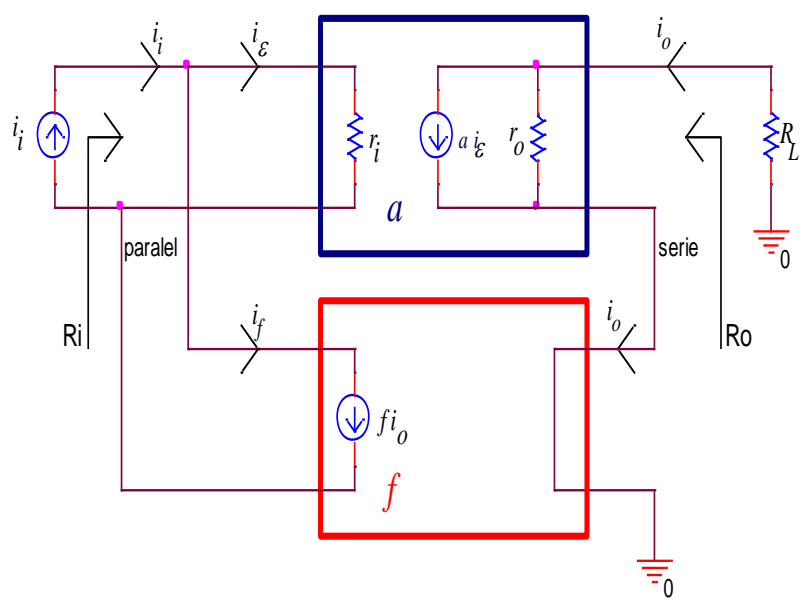

Figure 2. Complete parallel-series topology

$$
\begin{gathered}
i_{i}=i_{\varepsilon}+i_{f} \\
i_{\varepsilon}=i_{i}-i_{f} \\
r_{o} \gg R_{L} \Rightarrow i_{o}=a \cdot i_{\varepsilon} \Rightarrow i_{o}=a \cdot\left(i_{i}-i_{f}\right) \Rightarrow i_{o} \\
=a \cdot\left(i_{i}-f \cdot i_{o}\right) \\
\Rightarrow i_{o}=a \cdot i_{i}-a \cdot f \cdot i_{o} \Leftrightarrow i_{o}+a \cdot f \cdot i_{o}=a \cdot i_{i} \\
\Rightarrow i_{o}(1+a \cdot f)=a \cdot i_{i} \Rightarrow \frac{i_{o}}{i_{i}}=\frac{a}{1+a \cdot f} \Rightarrow A_{i} \\
=\frac{a}{1+a \cdot f}
\end{gathered}
$$

The input resistance is calculated with the diagram shown in Figure 2.

$$
\begin{gathered}
v_{i}=i_{\varepsilon} \cdot r_{i} \Rightarrow v_{i}=\left(i_{i}-i_{f}\right) \cdot r_{i} \Rightarrow v_{i}=\left(i_{i}-f \cdot i_{o}\right) \cdot r_{i} \\
\left.\begin{array}{rl}
v_{i}= & i_{i} \cdot\left(1-f \cdot \frac{i_{o}}{i_{i}}\right) \cdot r_{i} \\
\frac{i_{o}}{i_{i}}=\frac{a}{1+a \cdot f}
\end{array}\right\} \Rightarrow v_{i} \\
=i_{i} \cdot\left(1-f \cdot \frac{a}{1+a \cdot f}\right) \cdot r_{i}
\end{gathered}
$$




$$
\begin{gathered}
v_{i}=i_{i} \cdot\left(1-f \cdot \frac{a}{1+a \cdot f}\right) \cdot r_{i} \Rightarrow \frac{v_{i}}{i_{i}} \\
=\left(1-f \cdot \frac{a}{1+a \cdot f}\right) \cdot r_{i} \\
\left.\begin{array}{c}
\frac{v_{i}}{i_{i}}=\left(\frac{1+a \cdot f-a \cdot f}{1+a \cdot f}\right) \cdot r_{i} \Rightarrow \frac{v_{i}}{i_{i}}=\frac{1}{1+a \cdot f} \cdot r_{i} \Rightarrow \frac{v_{i}}{i_{i}} \\
=\frac{1}{1+T} \cdot r_{i} \\
\left.\frac{v_{i}}{i_{i}}=\frac{r_{i}}{1+T}\right) \\
\frac{v_{i}}{i_{i}}=R_{i}
\end{array}\right\} \Rightarrow R_{i}=\frac{r_{i}}{1+T}
\end{gathered}
$$

In order to determine the output resistance of the parallelseries topology, consider the circuit in Figure 3 where at the output instead of the load resistance a current source is introduced.

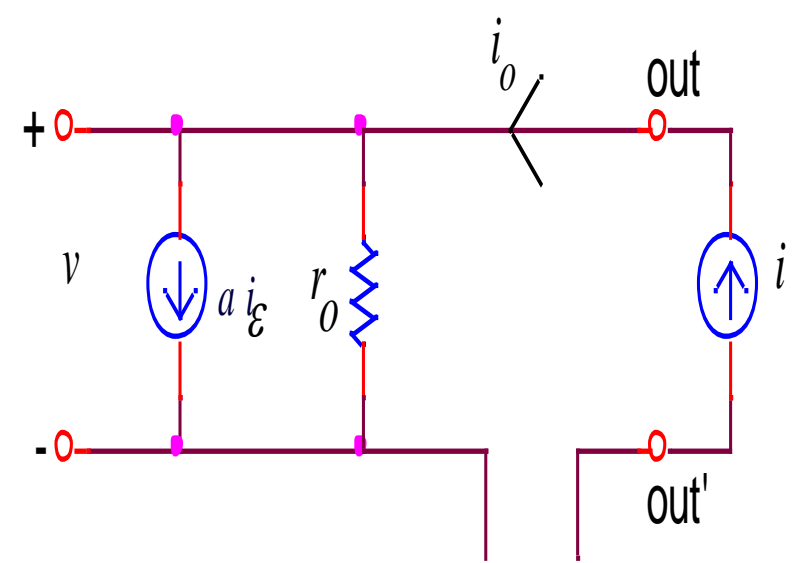

Figure 3. Circuit for calculating the output resistance

$$
\begin{aligned}
& R_{o}=\frac{v}{i} \\
& i_{i}=i_{\varepsilon}+i_{f} \\
& i_{\varepsilon}=-i_{f} \Rightarrow i_{\varepsilon}=-f \cdot i_{o}=-f \cdot i \text { pentru } i_{i}=0 \\
& v=\left(i-a \cdot i_{\varepsilon}\right) \cdot r_{o} \Rightarrow v=(i+a \cdot f \cdot i) \cdot r_{o} \Rightarrow v \\
& =i \cdot(1+a \cdot f) \cdot r_{o} \\
& \frac{v}{i}=(1+a \cdot f) \cdot r_{o} \Rightarrow \frac{v}{i}=(1+T) \cdot r_{o} \\
& \left.\begin{array}{c}
\frac{v}{i}=(1+T) \cdot r_{o} \\
\frac{v}{i}=R_{o}
\end{array}\right\} \Rightarrow R_{o}=r_{o} \cdot(1+T)
\end{aligned}
$$

\section{PARALLEL AMPLIFIER-SERIES}

In the case of the particular model, consider the diagram in Figure 4, in which the npn transistors Q1 and Q2 are characterized by $\beta_{F 1}=\beta_{F 2}=150, \beta_{01}=\beta_{02}=165$ and $V_{B E I}=$ $V_{B E 2}=0,65 \mathrm{~V}$. The values of the capacitors in the circuit are chosen so that, in alternating current, there is a short

circuit at the frequency of the signal provided by the generator (the amplifier works in the band).

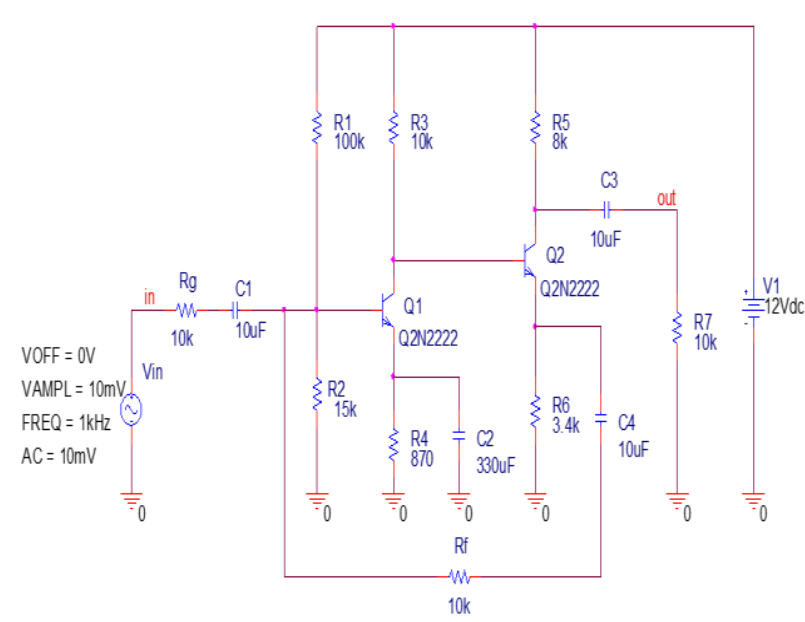

Figure 4. Parallel-series topology

\subsection{Parallel-series amplifier - direct current analysis}

A first step is to determine the static operating point (PSF). The effect of the negative reaction will be neglected in the direct current analysis. [3] Consider the diagram in Figure $5 \mathrm{a}$ in which the branches containing the capacitors were removed and $5 b$ where the Thévenin equivalence was performed.

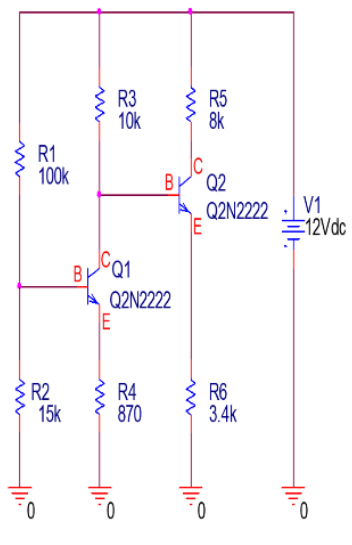

a).

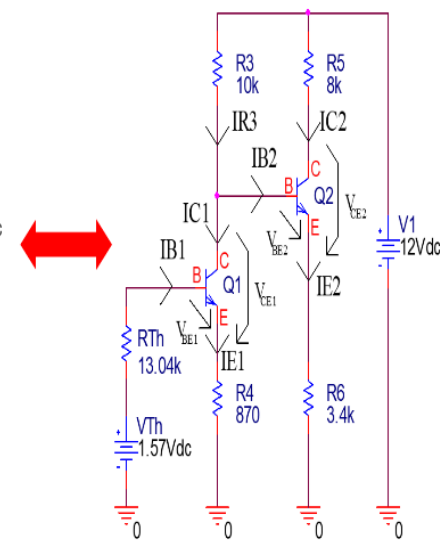

b).
Figure 5. DC analysis

$$
V_{T h}=\frac{R_{2}}{R_{1}+R_{2}} \cdot V_{1}=\frac{15 k \Omega}{115 k \Omega} \cdot 12 \mathrm{~V}=1,565 \mathrm{~V} \cong
$$

$1,57 \mathrm{~V}$

$$
R_{T h}=R_{1} \| R_{2}=\frac{R_{1} \cdot R_{2}}{R_{1}+R_{2}}=\frac{100 \mathrm{k} \Omega \cdot 15 \mathrm{k} \Omega}{115 \mathrm{k} \Omega}=13,043 \mathrm{k} \Omega
$$

Kirchhoff's theorem II is applied to the input eye (which contains the B-E junction of transistor Q1 - Figure. 5b) and to the output eye (which contains the C-E junction of transistor Q2 - Figure. 5b). 


$$
\begin{gathered}
V_{T h}=I_{B 1} \cdot R_{T h}+V_{B E 1}+I_{E 1} \cdot R_{4} \\
=I_{B 1} \cdot R_{T h}+V_{B E 1}+\left(\beta_{F 1}+1\right) I_{B 1} \\
\cdot R_{4}=I_{B 1} \cdot R_{T h}+\left(\beta_{F 1}+1\right) I_{B 1} \cdot R_{4} \\
V_{T h}-V_{B E 1}= \\
V_{T h}-V_{B E 1}=I_{B 1} \cdot\left[R_{T h}+\left(\beta_{F 1}+1\right) \cdot R_{4}\right] \\
I_{B 1}=\frac{V_{T h}-V_{B E 1}}{R_{T h}+\left(\beta_{F 1}+1\right) \cdot R_{4}}=\frac{1,57 V-0,65 \mathrm{~V}}{13,04 \mathrm{k} \Omega+(150+1) \cdot 0,87 \mathrm{k} \Omega}= \\
\frac{0,92 V}{144,41 k \Omega}=0,00637 \mathrm{~mA}(7) \\
I_{B 1} \cong 6,37 \mu \mathrm{A} \\
I_{C 1}=\beta_{F 1} \cdot I_{B 1} \Rightarrow I_{C 1}=150 \cdot 0,00637 \mathrm{~mA} \Rightarrow I_{C 1}= \\
0,9555 \mathrm{~mA} \quad(8) \\
I_{C 1} \cong 1 \mathrm{~mA} \\
I_{E 1}=\left(\beta_{F 1}+1\right) \cdot I_{B 1} \Rightarrow I_{E 1}=151 \cdot 0,00637 \mathrm{~mA} \Rightarrow \\
I_{E 1}=0,996187 \mathrm{~mA} \quad(9) \\
I_{E 1} \cong 1 \mathrm{~mA}
\end{gathered}
$$

We assume that: $I_{B 2} \ll I_{C 1}$

$$
I_{R 3}=I_{B 2}+I_{C 1} \Rightarrow I_{R 3} \cong I_{C 1} \cong 1 \mathrm{~mA}
$$

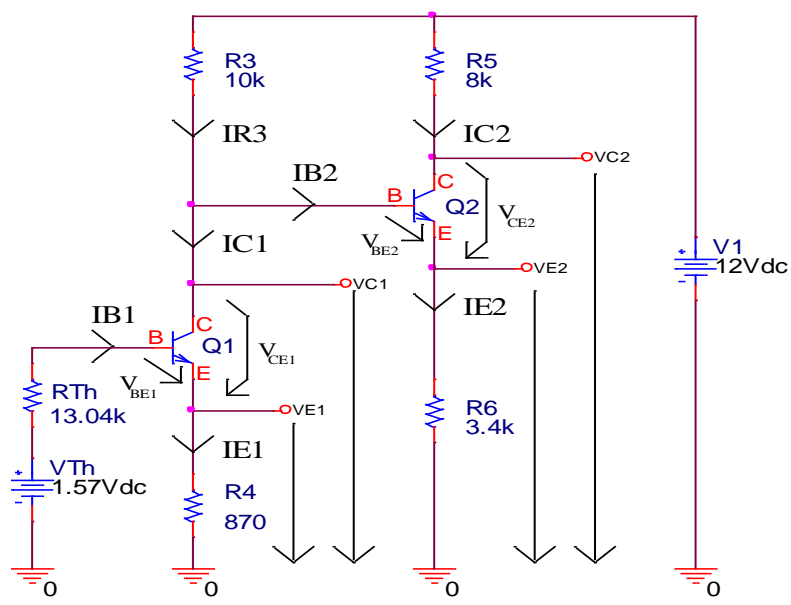

Figure 6. Direct current analysis, highlighting the potentials to Ground

$$
\begin{gathered}
V_{E 1}=I_{E 1} \cdot R_{4} \Rightarrow V_{E 1}=1 m A \cdot 0,87 k \Omega \Rightarrow V_{E 1}= \\
0,87 \mathrm{~V}
\end{gathered}
$$

Kirchhoff's theorem II is applied to the eye V1, R3, VC1.

$$
\begin{gathered}
V_{1}=I_{R 3} \cdot R_{3}+V_{C 1} \Rightarrow V_{C 1}=V_{1}-I_{R 3} \cdot R_{3} \Rightarrow \\
V_{C 1}=12 \mathrm{~V}-1 \mathrm{~mA} \cdot 10 \mathrm{k} \Omega(12) \\
V_{C 1}=12 \mathrm{~V}-10 \mathrm{~V} \Rightarrow V_{C 1}=2 \mathrm{~V}
\end{gathered}
$$

$$
V_{C E 1}=V_{C 1}-V_{E 1}=2 \mathrm{~V}-0,87 \mathrm{~V} \Rightarrow V_{C E 1}=1,13 \mathrm{~V}
$$

Kirchhoff's theorem II is applied to the eye VC1, VBE2, VE2.

$$
\begin{gathered}
V_{C 1}=V_{B E 2}+V_{E 2} \Rightarrow V_{E 2}=V_{C 1}-V_{B E 2} \Rightarrow V_{E 2}=2 V- \\
0,65 V \Rightarrow V_{E 2}=1,35 V(15) \\
V_{E 2}=I_{E 2} \cdot R_{6} \Rightarrow I_{E 2}=\frac{V_{E 2}}{R_{6}} \Rightarrow I_{E 2}=\frac{1,35 V}{3,4 k \Omega} \Rightarrow I_{E 2}= \\
0,397 m A \cong 0,4 m A(16) \\
I_{E 2}=\left(\beta_{F 2}+1\right) \cdot I_{B 2} \Rightarrow I_{B 1}=\frac{I_{E 2}}{\beta_{F 2}+1}=\frac{0,4 m A}{151}= \\
0,002649 m A \cong 0,00265 m A(17) \\
(18) \\
I_{B 2} \cong 2,65 \mu A \\
I_{C 2}=\beta_{F 2} \cdot I_{B 2} \Rightarrow I_{C 2}=150 \cdot 0,002649 m A \Rightarrow I_{C 2} \\
0,397 m A \cong 0,4 m A(19) \\
I_{C 2} \cong 0,4 m A
\end{gathered}
$$

It is verified that the assumption made is true: $I_{B 2}=$ $0,00265 \mathrm{~mA} \ll I_{C 1}=1 \mathrm{~mA}$

Kirchhoff's theorem II is applied to the eye V1, R5, VC2.

$V_{1}=I_{C 2} \cdot R_{5}+V_{C 2} \Rightarrow V_{C 2}=V_{1}-I_{C 2} \cdot R_{5} \Rightarrow V_{C 2}=$ $12 \mathrm{~V}-0,4 m A \cdot 8 k \Omega \quad(20)$

$$
\begin{gathered}
V_{C 1}=12 \mathrm{~V}-3,2 \mathrm{~V} \Rightarrow V_{C 1}=8,8 \mathrm{~V} \\
(21) \\
V_{C E 2}=V_{C 2}-V_{E 2}=8,8 \mathrm{~V}-1,35 \mathrm{~V} \Rightarrow V_{C E 2}=7,45 \mathrm{~V}
\end{gathered}
$$

The voltage $\mathrm{V}_{\mathrm{CE} 1}>\mathrm{V}_{\mathrm{BE} 1}$ and the voltage $\mathrm{V}_{\mathrm{CE} 2}>\mathrm{V}_{\mathrm{BE} 2}$, so $\mathrm{Q} 1$ and Q2 work in the normal active region (RAN).

$$
\begin{gathered}
\operatorname{PSF1}\left(I_{C 1}, V_{C E 1}\right) \Rightarrow P S F 1=\left\{\begin{array}{c}
I_{C 1}=1 m A \\
V_{C E 1}=1,13 \mathrm{~V}
\end{array}\right. \\
\operatorname{PSF2}\left(I_{C 2}, V_{C E 2}\right) \Rightarrow P S F 2=\left\{\begin{array}{c}
I_{C 2}=0,4 m A \\
V_{C E 2}=7,45 \mathrm{~V}
\end{array}\right.
\end{gathered}
$$

The values for the static point of operation (PSF) obtained by SPICE simulation are the following:

Table 1. Static point of operation (PSF)

\begin{tabular}{|c|c|c|}
\hline Name & Q_Q1 & Q_Q2 \\
\hline Model & Q2N2222 & Q2N2222 \\
\hline IB & $6.42 E-06$ & $3.53 E-06$ \\
\hline
\end{tabular}




\begin{tabular}{|c|c|c|}
\hline IC & $9.56 \mathrm{E}-04$ & $5.20 \mathrm{E}-04$ \\
\hline VBE & $6.44 \mathrm{E}-01$ & $6.27 \mathrm{E}-01$ \\
\hline VBC & $-9.27 \mathrm{E}-01$ & $-5.43 \mathrm{E}+00$ \\
\hline VCE & $1.57 \mathrm{E}+00$ & $6.06 \mathrm{E}+00$ \\
\hline BETADC & $1.49 \mathrm{E}+02$ & $1.47 \mathrm{E}+02$ \\
\hline
\end{tabular}

\subsection{Parallel-series amplifier - alternating current analysis}

To determine the small signal amplification, first we determine the small signal parameters:

Transconductance:

$$
\begin{gathered}
g_{m 1}=38 \cdot I_{C 1}=38 \cdot 1 \cdot 10^{-3}=38 m A / V \\
g_{m 2}=38 \cdot I_{C 2}=38 \cdot 0,4 \cdot 10^{-3}=15,2 m A / V
\end{gathered}
$$

Base-emitter resistance:

$$
\begin{aligned}
& r_{\pi 1}=\frac{\beta_{01}}{g_{m 1}}=\frac{150}{38 m A / V}=3,947 \frac{\mathrm{V}}{m A} \cong 3,95 \mathrm{k} \Omega \\
& r_{\pi 2}=\frac{\beta_{02}}{g_{m 2}}=\frac{150}{15,2 m A / V}=9,868 \frac{\mathrm{V}}{\mathrm{mA}} 2443,6 \Omega \cong 9,86 \mathrm{k} \Omega
\end{aligned}
$$

The AC analysis is done with the diagram in Figure 7, where capacitors $\mathrm{C} 1, \mathrm{C} 2, \mathrm{C} 3$ and $\mathrm{C} 4$ are considered shortcircuited. The DC voltage source, V1 is considered grounded.

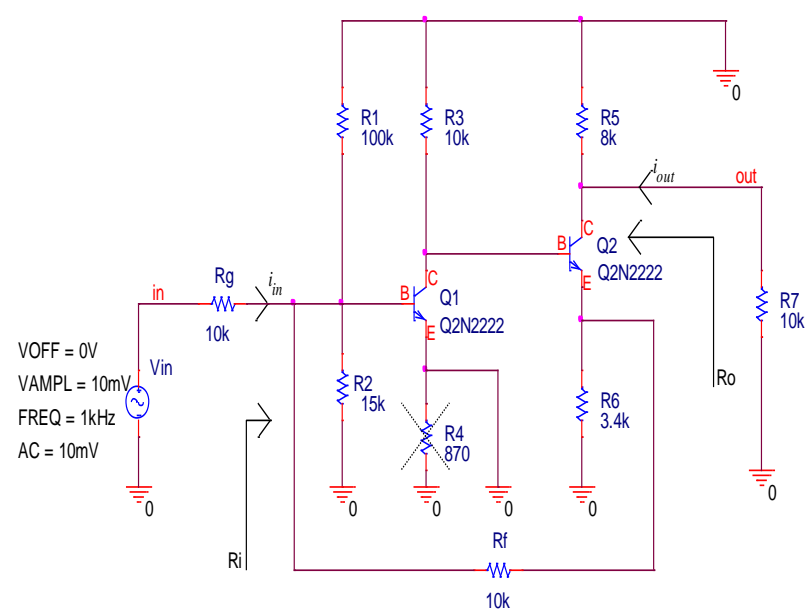

Figure 7. Alternating current analysis

The time response of the amplifier is shown in Figure 8, which shows that the output signal is $180^{\circ}$ out of phase with the input signal.

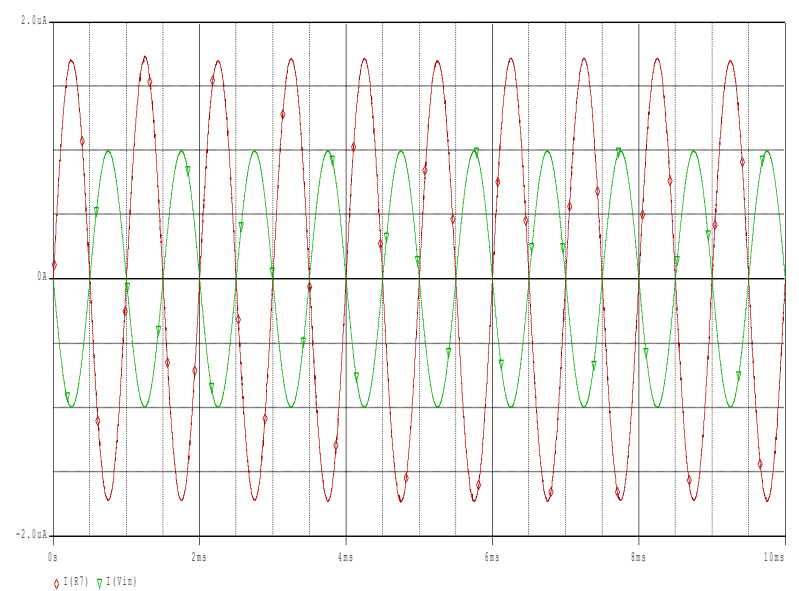

Figure 8. The time response of the amplifier

The equivalent small signal scheme is obtained by replacing the bipolar transistors with the simplified small signal model (pi-hybrid model) and the diagram in Figure 9 results:

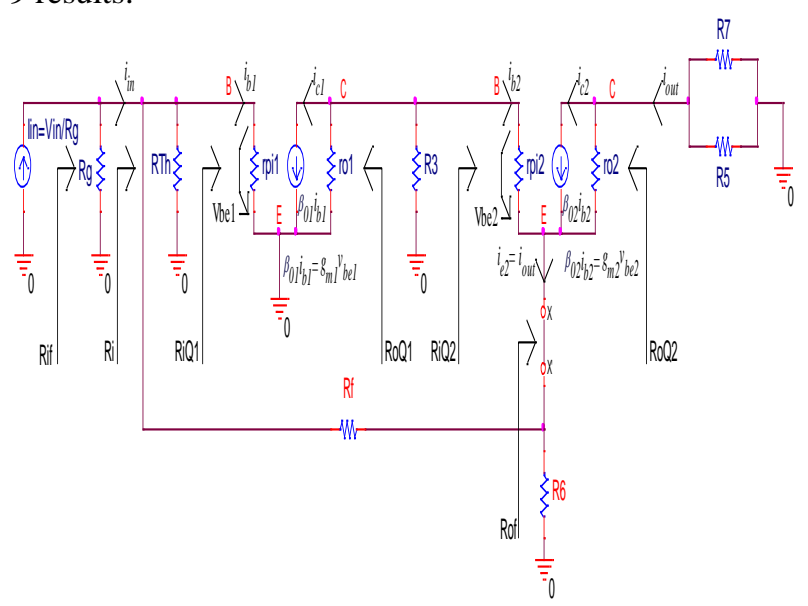

Figure 9. Equivalent small signal scheme

\subsubsection{Alternating current analysis - circuit a}

Circuit a is shown in Figure 10.

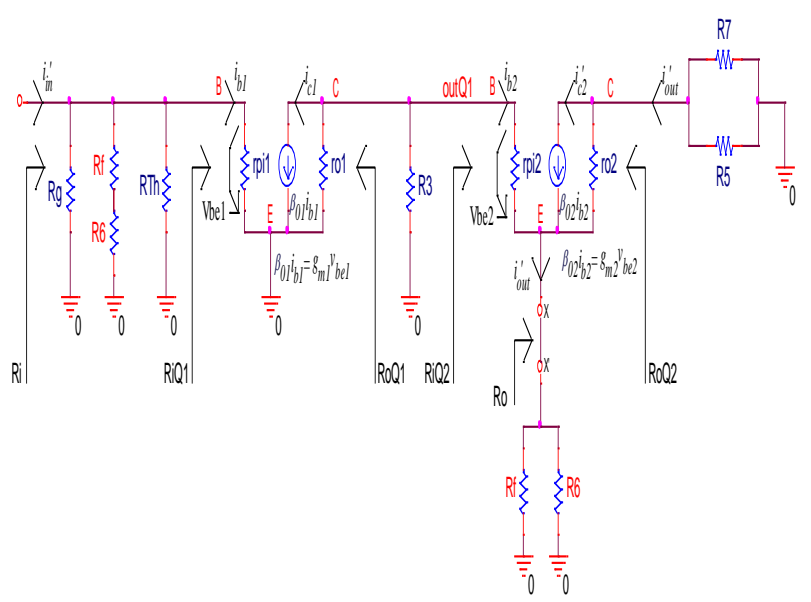

Figure 10. Equivalent small signal scheme for circuit a 


$$
\begin{aligned}
& R_{o Q 1} \rightarrow \infty \\
& R_{o Q 2} \rightarrow \infty
\end{aligned}
$$

It is calculated $v_{b e I}$.

$$
\begin{gathered}
v_{b e 1}=i_{i n}^{\prime} \cdot\left[R_{g}\left\|\left(R_{6}+R_{f}\right)\right\| R_{T h} \| R_{i Q 1}\right] \\
v_{b e 1}=i_{i n}^{\prime} \cdot\left[R_{g}\left\|\left(R_{6}+R_{f}\right)\right\| R_{T h} \| r_{\pi 1}\right] \\
i_{b 1} \cdot R_{i Q 1}=i_{b 1} \cdot r_{\pi 1} \Rightarrow R_{i Q 1}=r_{\pi 1} \\
i_{i n}^{\prime}=\frac{v_{b e 1}}{R_{g}\left\|\left(R_{6}+R_{f}\right)\right\| R_{T h} \| r_{\pi 1}}
\end{gathered}
$$

It is calculated $v_{\text {out } Q I}$.

$v_{\text {outQ1 }}=-i_{c 1} \cdot\left(R_{\text {oQ1 }}\left\|R_{3}\right\| R_{i Q 2}\right) \Rightarrow v_{\text {outQ1 }}=-g_{m 1}$. $v_{b e 1} \cdot\left(R_{3} \| R_{i Q 2}\right)(34)$

Calculation of the input resistance in Q2.

$$
\begin{gathered}
i_{b 2} \cdot R_{i Q 2}=i_{b 2} \cdot r_{\pi 2}+i_{e 2} \cdot\left(R_{6} \| R_{f}\right) \Rightarrow i_{b 2} \cdot R_{i Q 2} \\
=i_{b 2} \cdot r_{\pi 2}+i_{b 2} \cdot\left(\beta_{02}+1\right) \\
\cdot\left(R_{6} \| R_{f}\right) \\
i_{b 2} \cdot R_{i Q 2}=i_{b 2} \cdot\left[r_{\pi 2}+\left(\beta_{02}+1\right) \cdot\left(R_{6} \| R_{f}\right)\right] \\
(35) \\
R_{i Q 2}=r_{\pi 2}+\left(\beta_{02}+1\right) \cdot\left(R_{6} \| R_{f}\right)
\end{gathered}
$$

$$
\begin{gathered}
v_{\text {out } Q 1}=-g_{m 1} \cdot v_{b e 1} \cdot\left\{R_{3} \|\left[r_{\pi 2}+\left(\beta_{02}+1\right)\right.\right. \\
\left.\left.\left(R_{6} \| R_{f}\right)\right]\right\}(37)
\end{gathered}
$$

But

$$
\begin{aligned}
& v_{\text {outQ1 }}=i_{b 2} \cdot r_{\pi 2}+i_{\text {out }}^{\prime} \cdot\left(R_{6} \| R_{f}\right) \Rightarrow v_{\text {outQ1 }}=\frac{i_{c 2}}{\beta_{02}} . \\
& r_{\pi 2}+i_{\text {out }}^{\prime} \cdot\left(R_{6} \| R_{f}\right)(38) \\
& v_{\text {outQ1 }}=\frac{i_{\text {out }}^{\prime}}{\beta_{02}} \cdot r_{\pi 2}+i_{\text {out }}^{\prime} \cdot\left(R_{6} \| R_{f}\right) \Rightarrow v_{\text {outQ } 1} \\
& =i_{\text {out }}^{\prime} \cdot\left[\frac{1}{\beta_{02}} \cdot r_{\pi 2}+\left(R_{6} \| R_{f}\right)\right] \\
& i_{\text {out }}^{\prime}=\frac{v_{\text {out } Q 1}}{\frac{r_{\pi 2}}{\beta_{02}}+\left(R_{6} \| R_{f}\right)} \\
& i_{\text {out }}^{\prime}=\frac{-g_{m 1} \cdot v_{b e 1} \cdot\left\{R_{3} \|\left[r_{\pi 2}+\left(\beta_{02}+1\right) \cdot\left(R_{6} \| R_{f}\right)\right]\right\}}{\frac{r_{\pi 2}}{\beta_{02}}+\left(R_{6} \| R_{f}\right)}
\end{aligned}
$$

$$
a=\frac{i_{\text {out }}^{\prime}}{i_{\text {in }}^{\prime}}=\frac{\frac{-g_{m 1} \cdot v_{b e 1}\left\{\left\{R_{3} \|\left[r_{\pi 2}+\left(\beta_{02}+1\right) \cdot\left(R_{6} \| R_{f}\right)\right]\right\}\right.}{\frac{r_{\pi 2}}{\beta_{02}+\left(R_{6} \| R_{f}\right)}}}{\frac{v_{b e 1}}{R_{g}\left\|\left(R_{6}+R_{f}\right)\right\| R_{T h} \| r_{\pi 1}}}
$$

$a=\frac{-g_{m 1} \cdot v_{b e 1} \cdot\left\{R_{3} \|\left[r_{\pi 2}+\left(\beta_{02}+1\right) \cdot\left(R_{6} \| R_{f}\right)\right]\right\}}{\frac{r_{\pi 2}}{\beta_{02}}+\left(R_{6} \| R_{f}\right)} \cdot \frac{R_{g}\left\|\left(R_{6}+R_{f}\right)\right\| R_{T h} \| r_{\pi 1}}{v_{b e 1}}$

$$
\begin{aligned}
& a=\frac{-g_{m 1} \cdot\left\{R_{3} \|\left[r_{\pi 2}+\left(\beta_{02}+1\right) \cdot\left(R_{6} \| R_{f}\right)\right]\right\} \cdot\left[R_{g}\left\|\left(R_{6}+R_{f}\right)\right\| R_{T h} \| r_{\pi 1}\right]}{\frac{r_{\pi 2}}{\beta_{02}}+\left(R_{6} \| R_{f}\right)} \\
& a=-256,35
\end{aligned}
$$

Input resistor for circuit a:

$R_{i}=R_{g}\left\|\left(R_{6}+R_{f}\right)\right\| R_{T h}\left\|R_{i Q 1} \Rightarrow R_{i}=R_{g}\right\|\left(R_{6}+\right.$ $\left.R_{f}\right)\left\|R_{T h}\right\| r_{\pi 1}=0,504 k \Omega$

Output resistance for circuit a:

$R_{o}=\left(R_{6} \| R_{f}\right)+\frac{r_{\pi 2}}{\beta_{02}}+\frac{R_{3}}{\beta_{01}+1}=2,65 k \Omega$

\subsubsection{Alternating current analysis - circuit $f$}

Circuit $\mathrm{f}$ is shown in figure 11.

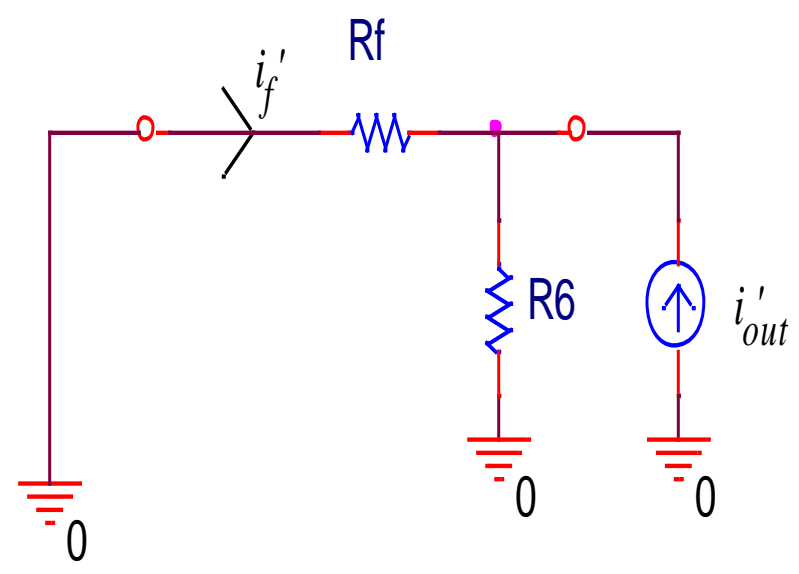

Figure 11. Equivalent small signal scheme for circuit $f$

The current divider rule applies

$$
\begin{gathered}
-i_{f}^{\prime}=\frac{R_{6}}{R_{6}+R_{f}} \cdot i_{\text {out }}^{\prime} \Rightarrow \frac{i_{f}^{\prime}}{i_{\text {out }}^{\prime}}=-\frac{R_{6}}{R_{6}+R_{f}} \\
f=\frac{i_{f}^{\prime}}{i_{\text {out }}^{\prime}}=-\frac{R_{6}}{R_{6}+R_{f}}=-\frac{3,4 k \Omega}{3,4 k \Omega+10 k \Omega}=-\frac{3,4 k \Omega}{13,4 k \Omega}= \\
-0,2537 \cong-0,254(45) \\
A_{i}=\frac{i_{\text {out }}}{i_{\text {in }}}=\frac{a}{1+a \cdot f}=\frac{-256,35}{1+(-256,35) \cdot(-0,254)}=-\frac{256,35}{66,113}= \\
-3,877(46)
\end{gathered}
$$


$R_{\text {if }}=\frac{R_{i}}{1+a \cdot f}=\frac{0,504 k \Omega}{1+(-256,35) \cdot(-0,254)}=\frac{0,504 k \Omega}{66,113}=$ $0,007632 k \Omega=7,62 \Omega \quad(47)$

$$
\begin{gathered}
R_{o f}=\frac{R_{o}}{1+a \cdot f}=\frac{2,65 k \Omega}{1+(-256,35) \cdot(-0,254)}=\frac{2,65 k \Omega}{66,113}= \\
0,04008 k \Omega=40,08 \Omega
\end{gathered}
$$

The frequency response of the amplifier is shown in Figure 12.

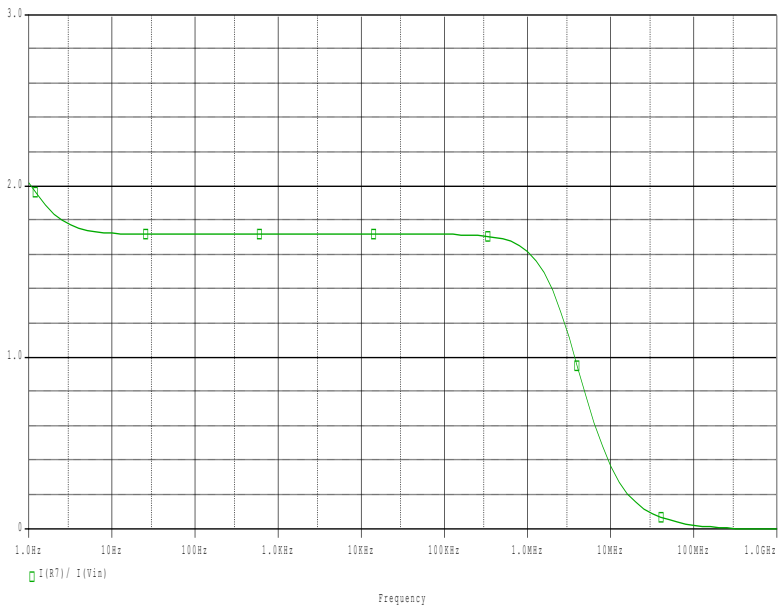

Figure 12. Frequency response of the amplifier

\section{CONCLUSIONS}

Circuits without a negative reaction are affected by the dispersion of parameters and disturbances. The use of the reaction helps to obtain an amplifier with a known amplification, impedances and convenient band. The choice of the reaction topology is chosen according to the wishes of the designer. If the chosen topology contains a series, one of the impedances does not benefit from the reaction advantage.

\section{REFERENCES}

[1] Eugen Stefan Lakatos, Dan Constantin Puchianu, Modelare și simulare în SPICE. Manual de laborator, Valahia University Press, Târgoviște, ISBN 978606-603-140-0, 2015.

[2] Eugen Stefan Lakatos, Dan Constantin Puchianu, Proiectare în microelectronică, Valahia University Press, Târgoviște, ISBN 978-606-603-179-0, 2017.

[3] E. Diaconu, "Controlul sistemelor electronice", Targoviste, Romania, Valahia University Press, ISBN 978-606-603-170-7.

[4] Dan Constantin Puchianu, Ion Caciula, Horia Andrei, Emil Diaconu, Paul Cristian Andrei, „New approaches of nonlinear circuit elements analysis in frequency domain", Electronics, Computers and
Artificial Intelligence International Conference ECAI 2017 -9th Edition, 29 June - 01 July, 2017, Târgoviste, ROMÂNIA, ISBN 978-1-5090-6458-8.

[5] Horia Andrei, Lucian Nastase, Emil Diaconu, Costin Cepisca, Sorin Dan Grigorescu, Paul Cristian Andrei, Contributions on Sensitivity Analysis for the Analog Two-Port Networks in Non-sinusoidal Regime, IEEE-EUROCON 2011 International Conference on Computer as a Tool - ConfTele 2011,27-29 April, 2011, Lisbon, Portugal, IEEE Catalog Number: CFP11EUR-CDR, ISBN 978-1-4244-7485-1, indexat IEEExplore/ISI Proceedings. 